25 Steinman, L., Lindsey, J.W., Alters, S. and Hodgkinson, S. (1993) in Monoclonal Antibody and Peptide Therapy in Autoimmune Diseases (Bach, J.F., ed.), pp. 253-260, Marcel Dekker

26 Strom, T.B., Kelley, V.R., Woodworth, T.G. and Murphy, J.R. (1992) Immunol. Rev. 129, 131-163

27 Rayner, D.C., Champion, B.R. and Cooke, A. (1993) in Monoclonal Antibody and Peptide Therapy in Autoimmune Diseases (Bach, J.F., ed.), pp. 359-375, Marcel Dekker

28 Maki, T., Ichikawa, T., Blanco, R. and Porter, J. (1992)

Proc. Natl Acad. Sci. USA 89, 3434-3438

29 Miller, A., Lider, O. and Weiner, H.L. (1991) J. Exp.

Med. 174, 791-798
30 Wraith, D.C., Smilek, D.E. and Webb, S. (1992) J. Autoimmunity 5 (Suppl. A), 103-113

31 Rothbard, J.B. and McDevitt, H.O. (1993) in T-cell

Directed Immunointervention (Bach, J.F., ed.), pp. 286-299, Blackwell Scientific Publications

32 Jacob, C.O., van der Meide, P.H. and McDevitt, H.O. (1987) J. Exp. Med. 166, 798-803

33 Debray-Sachs, M. et al. (1991) J. Autoimmunity 4, 237-248

34 Campbell, I.L., Kay, T.W.H., Oxbrow, L. and

Harrison, L.C. (1991) J. Clin. Invest. 87, 739-742

35 Jacob, C.O., Aiso, S., Michie, S.A., McDevitt, H.O. and Acha-Orbea, H. (1992) Immunology 87, 968-972

36 Bach, J.F. Ann. N. Y. Acad. Sci. (in press)

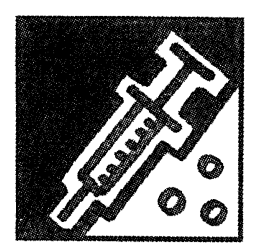

\title{
Donor cell chimerism permitted by immunosuppressive drugs: a new view of organ transplantation
}

\author{
Thomas E. Starzl, Anthony J. Demetris, \\ Noriko Murase, Angus W. Thomson, \\ Massimo Trucco and Camillo Ricordi
}

\begin{abstract}
One line of thought in organ transplantation feels that immunosuppressive drugs can lead to tolerance induction by allowing a previously unrecognized common mechanism of cell migration and microchimerism to occur, persist, and in some cases, become drug independent. It has been recognized that there is a spectrum of susceptibility of different organs to cellular rejection and that the variable ability of these organs to induce donorspecific nonreactivity reflects their comparative content of migratory leukocytes. Here, Thomas Starzl and colleagues discuss how many of the enigmas of transplantation immunology can be explained by this chimerism.
\end{abstract}

The prevention of organ rejection by various immunosuppressive agents has been described increasingly in terms of the molecular site of disruption of the alloactivated T-cell response ${ }^{1,2}$. Recent evidence, however, suggests that the control of rejection and, ultimately, graft acceptance depend on a permissive effect of these drugs on a mutual host-graft leukocyte migration that leads in successful cases to mixed, long-term microchimerism in the recipient as well as the transplant $^{3}$ (Fig. 1).

\section{An empiric drug regimen}

Observations compatible with this concept were reported in 1963 when combination therapy with azathioprine and prednisone was introduced for kidney transplantation ${ }^{4}$. There is a characteristic cycle of renal graft rejection in the first few days or weeks that can be reversed with steroids. The ability to reduce (Fig. 2), or sometimes even to stop, treatment was thereafter confirmed in cases of transplantation of the liver, heart and other organs.

The reproducibility of these events led to the fundamental therapeutic dogma that forms the basis of whole organ transplantation surgery. It calls for daily baseline treatment with a maintenance drug or drugs (originally azathioprine) plus trial and error intervention with the highly dose-manouverable adrenal cortical steroids, along with anti-lymphoid agents to whatever level is required to maintain stable graft function. Throughout the years, this policy framework has accommodated increasingly potent new agents with variable sites of action (Table 1).

\section{The chimeric host and graft}

Under the regimes described above it became evident that something, not drug specific, appeared to have changed in either the graft, the recipient or both. But what? 


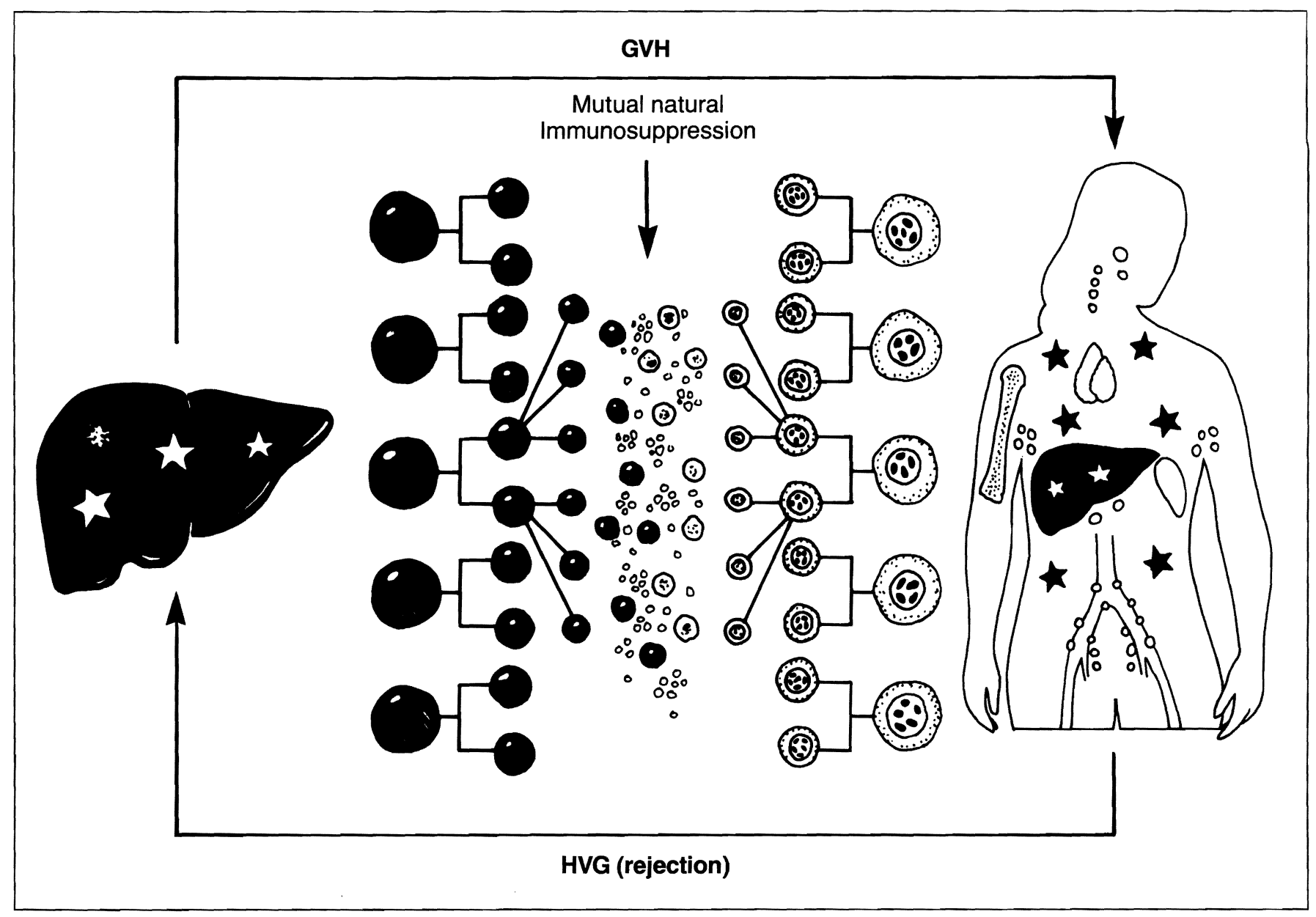

Fig. 1. The mutual engagement of migratory immunocytes from the graft and the recipient following organ transplantation under potent pharmacological immunosuppression. GVH: graft versus bost; HVG: host versus graft.

\section{Kidney transplantation}

A clue was found when tuberculin, coccidiodin and other delayed hypersensitivity skin test reactions in live kidney donors were shown to be transferred to the previously negative recipients, along with the transplanted kidneys ${ }^{5}$. Speculation that this was 'caused by adoptive transfer of donor cellular immunity by leukocytes in the renal graft vasculature and hilar lymphoid tissue', was undermined by the perception of the kidney as a leukocyte poor organ. Several years would pass before it became accepted that tissues and organs contained immunologically significant 'passenger leukocytes"7. It was subsequently demonstrated that these included the distinctive bone marrow-derived dendritic cells ${ }^{8}$ initially defined in 1973 by Steinman and Cohn ${ }^{9,10}$ that were correlated with organ immunogenicity ${ }^{11}$.

Confirmation that cell migration had occurred came when five of the original kidney transplant patients were reexamined in $1992^{12}$, after nearly 30 years. Their still functioning HLA mismatched kidneys were studied; HLA allele or Y chromosome analysis using cytochemical or polymerase chain reaction (PCR) techniques on biopsies of all five of the allografts showed that the interstitial leukocyte population was largely made up of recipient cells while the nephrons remained donor. More importantly, donor cells that appeared to be dendritic leukocytes were also found in the skin and lymph nodes of each of the five recipients. The presence of donor DNA in these locations was confirmed by PCR; blood chimerism was also detected in two of the five. For four patients the volunteer donors were still alive and all four showed donor-specific nonreactivity by mixed lymphocyte reactivity (MLR) and/or cell-mediated lymphocytotoxicity (CML) testing ${ }^{12}$.

\section{Liver and other organs}

Another under-appreciated clue to cell migration was the demonstration in 1969 that the Kupffer cells and other interstitial monocytes and macrophages of a transplanted human liver were replaced within 100 days by cells of the recipient phenotype ${ }^{13}$. The resulting composite (chimeric) structure was assumed to be a special feature of the hepatic graft. However, it was shown in 1991 that the transplanted intestine underwent a similar transformation in rats ${ }^{14}$ and humans ${ }^{15}$. It was therefore suspected that this process must be generic to all successfully engrafted organs; and was soon confirmed (reviewed in Ref. 16). The hematolymphoid nature of the responsible cell traffic was evident from the perioperative burst of donor mononuclear cells in the peripheral blood of human liver-intestinal or intestinal recipients ${ }^{15,17}$. The question of the fate of the donor cells departing the human intestinal graft went unanswered, but it was promptly shown by Murase $e t$ al. in rats that 


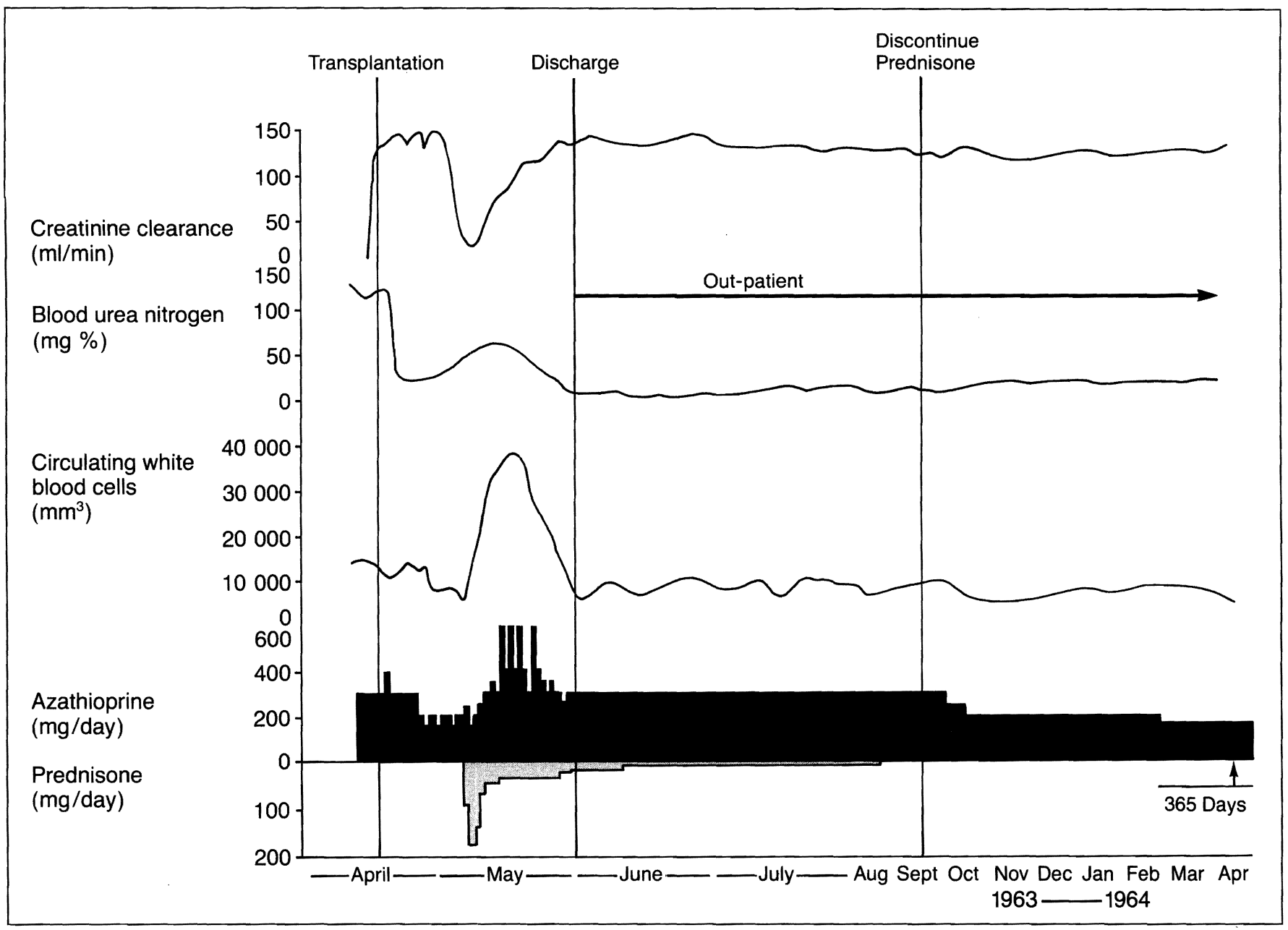

Fig. 2. The characteristic cycle of immunological confrontation and recovery after organ transplantation (in this case kidney) that has dictated the use of immunosuppressive drugs for the last 30 years. Following a period of good postoperative renal function, rejection 2-1/2 weeks after transplantation (Tx) was correlated with a fall in creatinine clearance (Ccr), a rise in blood urea nitrogen (BUN) and an increase in circulating white blood cells (WBC). The adverse findings were reversed with prednisone, which was later discontinued along with a reduction of maintenance azathioprine. The kidney allograft in this 23 year old man functioned continuously from April 1963 until death from a myocardial infarction in 1990. (Adapted from Starzl, T.E., Experience In Renal Transplantation, W.B. Saunders Company, Philadelphia, PA, 1964, p. 167.)

these cells from the intestine did not perish out to at least two months and in certain strain combinations uniformly caused graft versus host disease $(\mathrm{GVHD})^{18,19}$.

As with kidney transplantation, long-term chimerism after liver replacement was proved in 1992 by studying 25 patients who had received livers 2 to 22 years previously under treatment with azathioprine or cyclosporine-based immunosuppression ${ }^{3,16,20,21}$. Most were clinically well and fully immunocompetent as shown by conventional in vitro testing. Using the same cytochemical or PCR techniques as for the long surviving kidney recipients, donor cell chimerism was found in all 25 patients in locations that included skin, lymph nodes, heart, lungs, spleen, intestine, kidneys, bone marrow and thymus. Chimeric cells were found in larger numbers at any given site than in the contemporaneously studied long surviving kidney recipients. The heavy endowment of the liver with potentially migratory white cells was postulated to be the basis for the 'hepatic tolerogenicity' that allows the liver to induce its own acceptance more readily than other transplanted organs (in some experimental models without immunosuppression ${ }^{22-24}$. This is also postulated to be the basis by which donor livers shield concomitantly transplanted organs from rejection ${ }^{24,25}$, and even to resist the attack of preformed antibodies ${ }^{26}$.

\section{Cell augmentation with donor bone marrow, splenocytes} or blood

Thus, the hypothesis is that the heavy content (and perhaps the specific lineage) of migratory cells in the liver accounts for the immunological advantages of the hepatic graft. A corollary, therefore, is that organs such as the kidney and heart with a smaller leukocyte component must have similar inherent, although less tolerogenic, potential. The frequently advanced strategy of intravenously infusing donor bone marrow, donor blood (donor specific transfusion) or other hematolymphoid cells at the same time, or shortly after, transplantation of whole organs ${ }^{27-31}$ is merely an augmentation of the normal post-transplant cell migration. To mimic the natural process, these cells should be given perioperatively, not in advance or afterwards as usually has been done with the so-called Monaco model. 


\begin{tabular}{lll}
\hline Strategy & Baseline agents & Sites of action \\
\hline 1) Baseline therapy with one or two drugs & 1 Azathioprine & DNA synthesis \\
& 2 Cyclophosphamide & DNA synthesis \\
& 3 Cyclosporin & IL-2 production \\
2) Secondary adjustments with steroids or antilymphoid agents & 4 FK506 & IL-2 production \\
3) Case to case trial (and potential error) of weaning & & \\
\hline
\end{tabular}

Graft and host chimerism appears to be an invariable finding after (and we believe an essential condition for) successful organ transplantation. Indeed, it may become independent of drug therapy but there is no way presently to know if and when the time has arrived. In a recently reported group of 44 human liver recipients who had survived between 11 and 23 years, there were $6(14 \%)$ who had stopped all immunosuppression one to 11 years postoperatively with subsequent, clinically stable, drug-free intervals of between 5 and 13 years $^{16}$; another 15 with shorter follow-up periods are stable without drugs. The most extreme example of early successful drug discontinuance was after two months. A trial of drug weaning has been started in liver recipients with a rejection-free course exceeding five years. Liver graft rejection (if it occurs) can be so effectively treated with FK506 (Ref. 32) that the benefits of coming off drugs appear to us to outweigh the risks in selected patients.

The relative safety of drug stoppage in liver versus kidney recipients was shown long ago in dogs treated with azathioprine for only four postoperative months ${ }^{22}$. Canine liver recipients rarely rejected their grafts subsequently, whereas the incidence of fatal rejection of kidney allografts was $75 \%$ (Ref. 33). The higher risk from rejection after stopping immunosuppression in human kidney compared to liver recipients is well known. Nevertheless, a drug free state after renal allotransplantation has been accomplished more frequently than is generally known, particularly with the use of living related donors. Of the 17 kidney allografts in the world that are still continuously functioning from before March 1964, 10 are carried by the original Colorado patients in whom azathioprine-prednisone therapy was developed $^{34,35}$. Four of these 10 recipients (all with living, related kidneys) have been off drug treatment for 6 months, 12, 27 and 28 years. Strober et al..$^{36}$ have reported successful drug weaning in cadaver kidney recipients pretreated with total lymphoid irradiation and a short course of conventional postoperative drug therapy.

Functional consequences of microchimerism: cause and effect

Although the chimeric donor cells appear sparse in recipient tissues, even after liver transplantation, their widespread distribution suggests that the cumulative load must be substantial.

\section{Metabolic effects}

The striking ability of the minority population of chimeric cells to have widespread effects on body metabolism was shown in patients treated with liver transplantation for the pancellular enzyme deficiencies of Type 4 glycogen storage disease (branching enzyme deficiency with amylopectin storage) and Gaucher's disease ( $\beta$-glucocerebrosidase deficiency and storage of glucocerebroside $)^{21}$. Although these disorders were previously thought to be correctable only with bone marrow transplantation, there was dramatic resorption of both kinds of storage material from host extrahepatic tissues over periods of two to eight years after liver replacement. In these patients, donor cells could be demonstrated in the heart, lymph nodes, bone marrow, intestine, skin and elsewhere. There is apparently a coculture effect in these tissues of a small number of chimeric donor cells on the contiguous overwhelming numbers of enzyme-deficient recipient cells. This raises important questions about the potential cell-to-cell effect of other molecules directly involved in immunological processes, including tolerance induction.

\section{The immunological interface}

Much needs to be learned about how the chimeric donor cells, which resemble dendritic cells, are perpetuated for as long as three decades post-transplantation. At the outset, dendritic cells could be spawned by small numbers of progenitor cells in the residual blood or interstitium of the transplanted organ; such precursors have been grown from mouse blood, bone marrow, or whole organs using GM-CSF enriched media $^{37}$. More likely, the tissue leukocytes in the organ have not reached terminal differentiation as previously assumed, but are capable of immediate migration and cell division. Whatever the explanation of the early events, subsequent survival and renewal of these cells must depend upon chronic mutual stimulation of the donor and recipient cell populations. Bandeira et al. ${ }^{38}$ have emphasized that tolerance in this context shares many of the cellular characteristics associated with immunity. As to their immunological function, there is no direct way to test how this small population of donor dendritic and other cells could have an impact far exceeding its numbers.

\section{Changed host and graft interactions}

In spite of these limitations of measurement, it can be inferred that the coexisting immunocyte populations in successful cases come to regard each other in a revised light. The evidence on one hand is the fading of the threat of clinical rejection concomitant with development of donor specific reactivity in spite of lightened treatment, and on the other, the waning 
spectre of GVHD. Appreciation of this latter change has been the crucial factor permitting the successful engraftment of leukocyte-rich organs such as the liver, intestine, both together, or all of the intra-abdominal organs (multivisceral transplantation). The secrets were not to deplete the immunological component of the graft and not to alter the recipient reactivity with pretreatment ${ }^{39}$, and to accept that mixed allogeneic chimerism was a natural consequence of organ transplantation under immunosuppression. No leap of faith was then required to equate the GVHD resistance seen in the liver, intestinal, or multivisceral recipient to the GVHD resistance described years ago in mouse bone marrow transplant models of mixed chimerism by Slavin and Strober ${ }^{28}$ and Ildstad and Sachs ${ }^{29}$.

\section{Impact on tissue matching}

In both the directions of host versus graft (HVG) and graft versus host $(\mathrm{GVH})$, cellular interactions resulting in 'mutual natural immunosuppression' are envisioned as occurring on a sliding scale with each further level of histoincompatibility (Fig. 3). Under the protective umbrella of modern day immunosuppression, the acute storm can be weathered long enough to allow the changes caused by the cell engraftment to occur and a rapprochement to be reached. The anticipated histocompatibility influence on both rejection and the severity of GVHD are then expected to dwindle and this may explain the poor correlation of HLA matching with outcome in whole organ cadaveric transplantation.

\section{Migratory cell load and pace of donor-recipient change}

The rapidity and/or ease of this transformation is undoubtedly increased by a heavy load of the tolerogenic leukocytes migrating naturally from a transplanted organ (re: liver transplantation) or by a supplemental infusion of bone marrow or blood (see earlier section). However, the mutual drug-free 'take' of the leukocytes cannot be made time independent by cell dosage only. The rapidly evolving drug free donor specific tolerance that comes with multilineage, mixed allogeneic or xenogeneic bone marrow chimerism in small rodents ${ }^{28,29,40}$ has created an unrealistic expectation that the same thing applies for outbred major histocompatibility complex (MHC) mismatched large animals and humans.

Clinical trials with bone marrow supplementation for liver ${ }^{41}$ and kidney transplantation ${ }^{31}$ have shown that the guidelines for drug weaning under the circumstances of cell augmentation will be similar to those already established after conventional liver transplantation. Some patients may not need drug treatment after a few weeks or months to ensure stability of the mixed chimerism, some may require years and a few may never reach this state without risking rejection, GVHD or both.

\section{The critical dendritic cell}

Generation of an immune response leading, under normal circumstances, to graft destruction and/or GVHD requires effective antigen presentation and recognition in its initial phase followed by a second co- stimulatory signal and the response of $\mathrm{T}$ cells to the combined signal ${ }^{42}$. Both of these signals are normally delivered to $T$ cells by professional antigen-presenting cells (APC). Of these APCs, the dendritic cell (the most prominent chimeric cell by morphological criteria) is critical because it can modify the expression of cell interaction, MHC, and adhesion molecules - all of which determine how antigen signals are heeded by $T$ cells ${ }^{43}$. Thus the dendritic leukocyte is the prime candidate in this tolerogenicity scenario, even though other lineages may also be essential for the successful outcome of such an immunological transaction.

\section{Relation of cell migration to tolerance}

Recent reviews have emphasized the inadequacy of thymic clonal deletion to explain acquired transplantation tolerance and have focused on post-thymic mechanisms that include peripheral clonal deletion and anergy ${ }^{44}$.

\section{Compatibility with other hypotheses}

Although a discussion of the meaning of tolerance is beyond our intention, we note here that all of the hypotheses to explain 'Clonal silencing' will be enriched by the discovery of the enduring intimacy of the graft and host immunological systems that is inherent with chimerism. The evidence of vitality and turnover of donor leukocytes in recipient tissues as long as three decades post-transplantation is particularly supportive of the opinions of Coutinho ${ }^{38,45}$ and Cohen ${ }^{46}$. They have defined acquired tolerance as a high (not anergic) level of sustained immune activity in networks interacting in a more complex fashion than the idiotype systems originally postulated by Jerne ${ }^{47}$. Suppressor and/or veto cells could be epiphenomena of this kind of activity; perhaps it is not too much to ask if these are altered dendritic cells rather than $T$ lymphocytes, as commonly assumed.

Throughout the years, the testing of every genuinely potent immunosuppressant has been followed by excited claims of tolerance induction. Now a common mechanism appears to be cell migration and chimerism, no matter what the site of the drug action. The very simplicity of these events in organ recipients cloaked their existence and delayed their discovery. With this understanding, it should be possible using drugs with known sites of action to ask more specific questions about the relation of drug-induced tolerance to the kind of acquired tolerance originally produced with intrauterine or neonatal splenocyte inoculation in mice by Billingham, Brent and Medawar ${ }^{48}$.

For example, it has been proposed from observations in drug free models of tolerance induction that T-cell receptor (TCR) occupancy leads to production of negative regulators of IL-2 production (anergy proteins $)^{42,49}$. According to this hypothesis, during the course of a normal $\mathrm{T}$-cell response (to alloantigens) these negative regulators of IL-2 production have an inconsequential effect because they are diluted out by vigorous cell replication driven by IL-2. However, these negative regulators would accumulate with consequent anergy if clonal expansion were prevented at any level: for instance, by the absence of a costimulatory 
signal in drug-free models ${ }^{42}$ but also by pharmacological interdiction of IL-2 gene transcription (cyclosporin and FK506) or administration of a DNA synthesis inhibitor (azathioprine, cyclophosphamide and numerous others). The use of non T-cell depleting monoclonal antibodies, such as those directed against the cell surface CD4 antigen or monoclonal antibodies against adhesion molecules including ICAM-1 and LFA- $1^{50}$ can also be envisaged.

\section{Parking experiments revisited}

In the two-stage parking models ${ }^{11,40}$, stage 1 consists of the induction with drug or irradiation treatment of permanent whole organ acceptance, for which the word tolerance usually is studiously avoided, even though it would be an apposite term in the context of the cell migration hypothesis. Once the graft's passenger leukocytes are replaced by migratory cells from the host, the composite organ that is excised and retransplanted at the second stage is rejected by naive animals of the donor, but not the recipient strain. However, these results can be accomplished only when immunologically 'easy' rat strain combinations are used ${ }^{11,40}$ or with perfect MHC matching in larger animals ${ }^{51}$. Even then, the outcome tends to be variable.

The parking model has been a useful research tool. However, results from parking experiments cannot be freely extrapolated to a discussion of the cell migration concept because neither the host immunocytes (including those that home to the parked organ) nor the donor leukocytes seeded ubiquitously in the recipient remain the same. Throughout this discourse and elsewhere ${ }^{3,12,16,20,21}$, we have emphasized that the nonresponsiveness induced after cell migration involves $\mathrm{GVH}$ as well as host versus graft (HVG) reactions. The reciprocal educational process of donor and recipient leukocytes and its perpetuation resembles in either direction the 'infectious' transplantation tolerance that can be passed on to naive lymphocytes and be self sustaining in some circumstances (see $\mathrm{H}$. Waldmann and S. Cobbold, this issue, pp. 247-251). In fully successful cases, the mini-immune system of the graft is incorporated into the existing recipient immunological network, compatible with the hypothesis of Coutinho ${ }^{45}$. Incompleteness of this assimilation on the HVG limb (Fig. 1) is monitored by evidence of rejection, which has been the sole measurable end-point of all parking experiments.

Assimilation on the GVH limb also is ordinarily silent, but it can be unmasked with transplantation experiments using the LEW to $\mathrm{BN}$ strain combination that is inherently GVHD prone ${ }^{18,19}$. The experiments consisted of simulating with a bone marrow infusion the natural cell migration that occurs following whole organ transplantation; then or later the migratory passenger leukocyte load brought in with a contemporaneous or delayed liver or heart allograft was added (Demetris, A.J. et al., submitted). In these rat experiments, liver transplantation plus donor strain bone marrow did not cause GVHD when both engraftments were done simultaneously under immunosuppression. However, when chimerism was induced with preliminary bone marrow transplantation followed by a 28

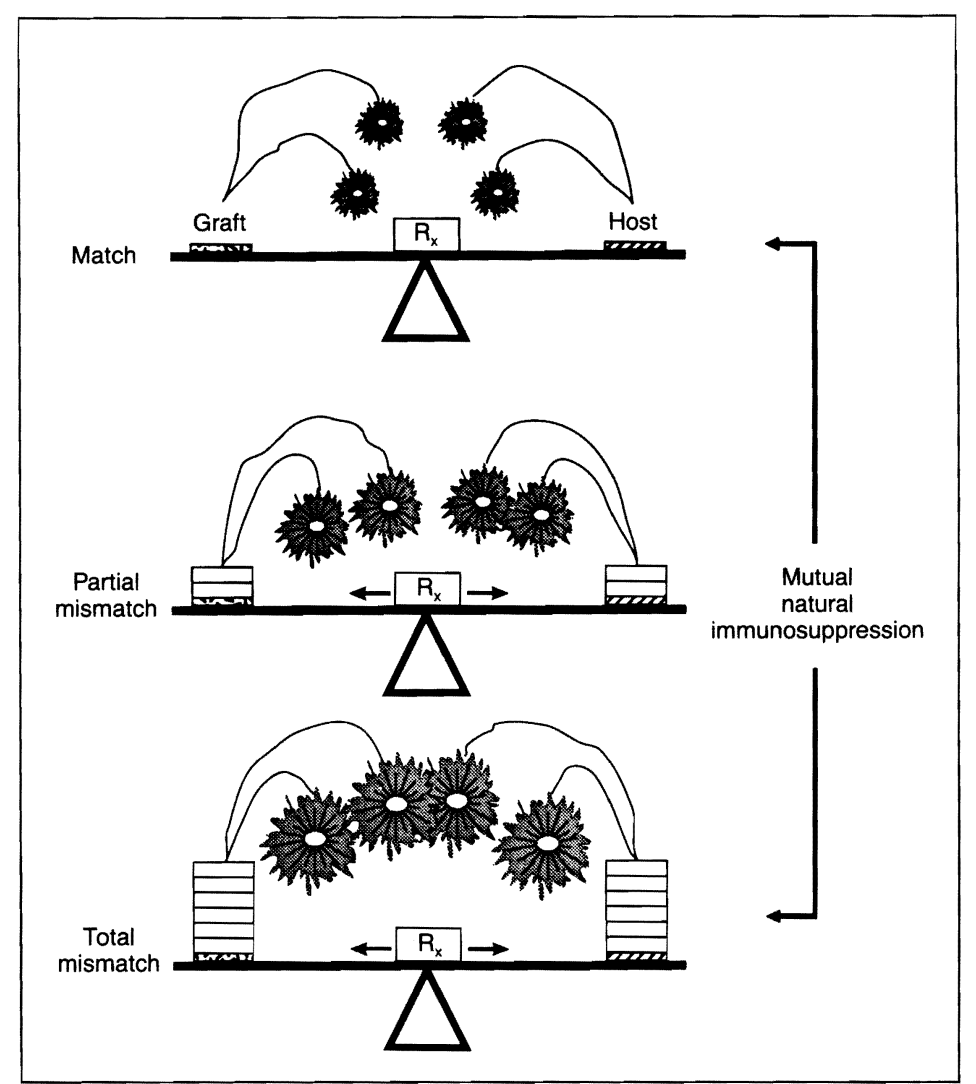

Fig. 3. The donor-recipient leukocyte interaction shown in Fig. 1 is a buffer against rejection on one hand and GVHD on the other. Veto and suppressor cells are postulated to be the result of the interaction. $R x$ : iatrogenic immunosuppression.

day course of FK506, subsequent liver transplantation after a drug free interval of 18 days invariably caused lethal GVHD.

The outcome with the delayed hepatic transplantation resembled that of a parent to offspring $F_{1}$ hybrid experiment in that the liver including its virgin migratory cells was seen as self by the altered host immune system, but not having gone through the process of modification, the hepatic passenger leukocytes reciprocated by rejecting the defenseless recipient. In contrast, heterotopic hearts transplanted under the same circumstances of prior bone marrow preparation were accepted without causing clinical GVHD, presumably reflecting the smaller supply of cardiac passenger leukocytes.

\section{Unstable mixed chimerism}

Because it inter-relates tolerance, rejection and GVHD, the cell migration concept creates a seamless single world for transplantation of bone marrow and of whole organs. Far from involving different mechanisms, we believe that these two seemingly disparate fields merely reflect contrasting treatment dogmas. For bone marrow transplantation, the treatment strategy of recipient cytoablation eliminates mutual immunocyte engagement and thus necessitates heavy reliance on HLA matching to prevent GVHD. The treatment for solid organ transplantation encourages, or at least permits, mutual cell engagement, thereby liberating the 
patient from the restrictions of HLA matching and an overwhelming threat of GVHD.

Failure of clinical organ transplantation implies the inability to achieve mixed allogeneic chimerism despite the best available immunosuppression, most commonly because of an imbalance leading to rejection. However, because an incipient GVH reaction is a necessary condition for success (this is our fundamental premise), clinical GVHD is a theoretical possibility after every transplantation, although the threat varies from organ to organ. We now know that about $5 \%$ of all liver recipients go through a bout of clinical GVHD that in the past usually was attributed to an allergic skin reaction ${ }^{16}$.

Although the complications of GVHD usually can be managed with an increase (most commonly) or decrease of immunosuppression, reports of a fatal outcome are not uncommon. In one case, the novel strategy of reinfusing a patient's stored bone marrow dramatically reduced the degree of chimerism and controlled life threatening GVHD that had developed six weeks after combined liver and bone marrow transplantation ${ }^{41}$. It is not known how the autologous naive cells that had been in storage differed from the immunocytes in the patient that had become defenseless against attack after cohabiting with chimeric donor cells but the clinical case appeared to be a 'mirror image' of the staged bone marrow-liver transplantation in rats described in the preceding section in which GVHD was switched on, not off. This therapeutic 'rescue' on the patient was reminiscent of a tolerance breaking experiment by infusion of a naive recipient strain of immunocytes that was described in 1956 by Billingham, Brent and Medawar ${ }^{48}$.

Guidance was generously and frequently provided by Robert A. Good (St Petersburg), G.J.V. Nossal (Melbourne), Ralph Steinman (New York), and Leslie Brent (London). During the preparation of the manuscript Nossal emphasized the similarity of our clinical observations to the phenomenon of 'exhaustive clonal differentiation' studied in the nontransplant mouse model ${ }^{52}$. Aided by project grant No DK29961 from the National Institutes of Health, Bethesda, Maryland.

Thomas Starzl, Angus Thomson, Noriko Murase and Camillo Ricordi are at the Dept of Surgery, University of Pittsburgh Health Science Center, Pittsburgh, PA 15213, USA; Anthony J. Demetris is at the Dept of Pathology, University of Pittsburgh Health Science Center, Pittsburgh, PA 15213, USA and Massimo Trucco is at the Dept of Pediatrics, University of Pittsburgh Health Sciences Center, Pittsburgh, PA 15213, USA.

\section{References}

1 Sigal, N.H. and Dumont, F.J. (1992) Annu. Rev. Immunol. 10, 519-560

2 Thomson, A.W. and Starzl, T.E. (eds) Immunosuppressive Drugs: Developments in Anti-Rejection Therapy, Edward Arnold, London (in press)

3 Starzl, T.E. et al. (1992) Lancet 339, 1579-1582

4 Starzl, T.E., Marchioro, T.L. and Waddell, W.R. (1963)

Surg. Gynecol. Obstet. 117, 385-395

5 Kirkpatrick, C.H., Wilson, W.E.C. and Talmage, D.W.

(1964) J. Exp. Med. 119, 727-742

6 Wilson, W.E.C. and Kirkpatrick, C.H. (1964) in

Experience In Renal Transplantation (Starzl, T.E., ed.), pp. 239-261, W.B. Saunders

7 Steinmuller, D. (1967) Science 158, 127-129

8 Hart, D.N.J. and Fabre, J.W. (1981) J. Exp. Med. 154, 347-361

9 Steinman, R.M. and Cohn, Z.A. (1973) J. Exp. Med. 137, 1142-1162

10 Steinman, R.M., Lustig, D.S. and Cohn, Z.A. (1974)

J. Exp. Med. 139, 1431-1445

11 Lechler, R.I. and Batchelor, J.R. (1982) J. Exp. Med.

$155,31-41$

12 Starzl, T.E. et al. Transplantation (in press)

13 Kashiwagi, N., Porter, K.A., Penn, I., Brettschneider, L. and Starzl, T.E. (1969) Surg. Forum 20, 374-376

14 Murase, N. et al. (1991) Surgery 110, 87-98

15 Iwaki, Y. et al. (1991) Lancet 337, 818-819

16 Starzl, T.E. et al. Hepatology (in press)

17 Grant, D. et al. (1990) Lancet 335, 181-184

18 Murase, N. et al. (1991) Transplant Proc. 23, 3246-3247

19 Murase, N. et al. (1993) Transplantation 55, 1-7

20 Starzl, T.E. et al. (1992) Lancet 340, 876-877

21 Starzl, T.E. et al. (1993) New Engl. J. Med. 328, 745-749

22 Starzl, T.E. et al. (1965) Surgery 58, 131-155

23 Garnier, H., Clot, J. and Bertrand, M. (1965) C. R.

Acad. Sci. Paris 260, 5621-5623

24 Calne, R.Y. et al. (1969) Nature 223, 472-474

25 Starzl, T.E., Kaupp, H.A., Jr, Brock, D.R., Butz, G.W., Jr and Linman, J.W. (1962) Am. J. Surg. 103, 219-229

26 Starzl, T.E. et al. (1974) Transplant Proc. 6, 129-139

27 Monaco, A.P. and Wood, M.L. (1970) Transplant Proc. $2,489-496$

28 Slavin, S., Strober, S., Fuks, Z. and Kaplan, H.S. (1977)

J. Exp. Med. 146, 34-48

29 Ildstad, S.T. and Sachs, D.H. (1984) Nature 307, 168-170

30 Thomas, J., Carver, M., Foil, B., Haisch, C. and Thomas, F. (1983) Transplantation 36, 104-106

31 Barber, W.H. et al. (1991) Transplantation 51, 70-75

32 Starzl, T.E. et al. (1989) Lancet ii, 1000-1004

33 Starzl, T.E. (1969) in Experience in Hepatic

Transplantation, pp. 203-207, W.B. Saunders

34 Graver, B. (1991) in Clinical Transplants (Terasaki, P.I. and Cecka, J.M., eds), pp. 431-434, UCLA Press

35 Starzl, T.E. et al. (1990) Transplant Proc. 22, 2361-2365

36 Strober, S. et al. (1989) New Engl. J. Med. 321, 28-33

37 Inaba, K. et al. (1992) J. Exp. Med. 176, 1693-1702

38 Bandeira, A., Coutinho, A., Carnaud, C., Jacquemart, F. and Forni, L. (1989) Proc. Natl Acad. Sci. USA 86, 272-276 39 Starzl, T.E. et al. (1991) Surg. Gynecol. Obstet. 172, 335-344

40 Gundlach, M., Oluwole, S., D’Agati, V., Chabot, J., Broellsch, C.E. and Hardy, M.A. (1992) Transplant Proc. 24, 1121-1132

41 Ricordi, C. et al. (1993) Transplant Science 3, 73-74

42 Jenkins, M.K. (1992) Immunol. Today 13, 69-73

43 Steinman, R.M. (1991) Annu. Rev. Immunol. 9, 271-296

44 Miller, J.F.A.P. and Morahan, G. (1992) Annu. Rev.

Immunol. 10, 51-69

45 Coutinho, A. (1989) Immunol. Rev. 110, 63-87

46 Cohen, I.R. (1992) Immunol. Today 13, 490-494

47 Jerne, N.K. (1984) Immunol. Rev. 79, 5-24

48 Billingham, R., Brent, L. and Medawar, P. (1956) Philos.

Trans. R. Soc. Lond. 239, 357-412

49 Zubiaga, A.M., Munoz, A. and Huber, B.T. (1991)

J. Immunol. 146, 3857-3863

50 Isobe, M., Yagita, H., Okumura, K. and Ihara, A. (1992)

Science 255, 1125-1127

51 Rapaport, F.T. et al. (1988) Transplantation 45, 682-686

52 Webb, S., Morris, C. and Sprent, J. (1990) Cell 63,

$1249-1256$ 